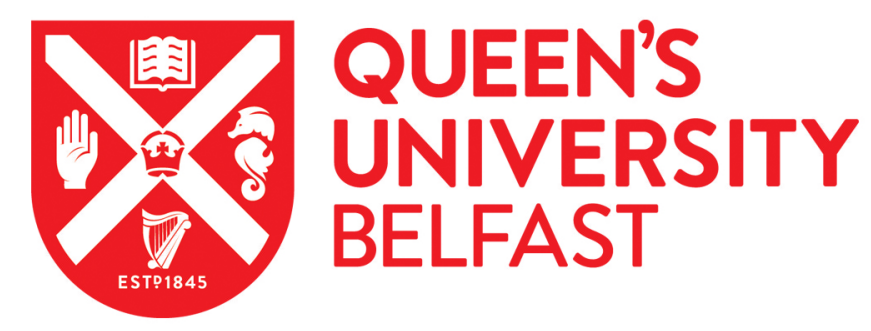

\title{
Improving Detection And Recognition Of Degraded Faces By Discriminative Feature Restoration Using GAN
}

Ghosh, S. S., Hua, Y., Mukherjee, S. S., \& Robertson, N. M. (2020). Improving Detection And Recognition Of Degraded Faces By Discriminative Feature Restoration Using GAN. In 2020 IEEE International Conference on Image Processing (ICIP): Proceedings (pp. 2146-2150). (IEEE International Conference on Image Processing (ICIP): Proceedings). Institute of Electrical and Electronics Engineers Inc.. https://doi.org/10.1109/ICIP40778.2020.9191246

Published in:

2020 IEEE International Conference on Image Processing (ICIP): Proceedings

Document Version:

Peer reviewed version

Queen's University Belfast - Research Portal:

Link to publication record in Queen's University Belfast Research Portal

Publisher rights

Copyright 2020 IEEE. This work is made available online in accordance with the publisher's policies. Please refer to any applicable terms of use of the publisher.

\section{General rights}

Copyright for the publications made accessible via the Queen's University Belfast Research Portal is retained by the author(s) and / or other copyright owners and it is a condition of accessing these publications that users recognise and abide by the legal requirements associated with these rights.

Take down policy

The Research Portal is Queen's institutional repository that provides access to Queen's research output. Every effort has been made to ensure that content in the Research Portal does not infringe any person's rights, or applicable UK laws. If you discover content in the Research Portal that you believe breaches copyright or violates any law, please contact openaccess@qub.ac.uk. 


\title{
IMPROVING DETECTION AND RECOGNITION OF DEGRADED FACES BY DISCRIMINATIVE FEATURE RESTORATION USING GAN
}

\author{
Soumya Shubhra Ghosh ${ }^{\star \dagger} \quad$ Yang Hua $_{\text {Sankha Subhra Mukherjee }}^{\dagger} \quad$ Neil M. Robertson ${ }^{\star \dagger}$ \\ ${ }^{\star}$ EEECS/ECIT, Queen’s University Belfast, ${ }^{\dagger}$ Anyvision
}

\begin{abstract}
Face detection and recognition in the wild is currently one of the most interesting and challenging problems. Many algorithms with high performance have already been proposed and applied in real-world applications. However, the problem of detecting and recognising degraded faces from low-quality images and videos mostly remains unsolved. In this paper, we present an algorithm capable of recovering facial features from low-quality videos and images. The resulting output image boosts the performance of existing face detection and recognition algorithms. It contains an effective method involving metric learning and different loss function components operating on different parts of the generator. This enhances the degraded faces by restoring their lost features rather than its perceptual quality. Our approach has been experimentally proven to enhance face detection and recognition, e.g., the face detection rate is improved by $3.08 \%$ for S3FD [1] and the area under the ROC curve for recognition is improved by $2.55 \%$ for ArcFace [2] on the SCFace dataset.
\end{abstract}

Index Terms - CNN, GAN, detection, recognition, loss function, degraded face image

\section{INTRODUCTION}

Face detection and recognition from low-quality images are challenging but highly demanded in real-world applications. These problems are particularly prominent for highly compressed images and videos, where details are typically absent. This is the most common in surveillance videos where the camera sensors are not of very high quality. Good detection and recognition performance are highly desirable in these scenarios and we have explored this possibility in this paper.

Solving the problem of detecting/recognising faces with high accuracy, different approaches have been proposed [1, 2, $3,4,5,6]$. Most recognition algorithms attempt to minimise the distance between similar identities and maximise between dissimilar identities, thus maximising the overall face recognition performance. The limitation of these methods is that their performance drops significantly with degraded images (e.g., for SCFace dataset) as seen in Figure 3).

Pixel-wise loss functions like MSE or L1 loss are unable to capture the perceptually relevant high-frequency image de- tails and encourage finding pixel-wise averages of possible solutions. This alone neither leads to the reconstruction of discriminative facial features nor leads to better perceptual quality [7, 8, 9, 10]. Johnson et al. [9] and Bruna et al. [7] proposed a perceptual loss function based on the Euclidean distance between feature maps extracted from the VGG19 [11] network. Zhang et al. proposed an algorithm [12] to solve different problems related to image artifacts. A recent paper by Ghosh et al. [13] uses edge loss on top of perceptual loss to simultaneously remove artifacts and super-resolve images. A major drawback of these algorithms is that they are not suitable for application when it comes to face detection or recognition. Improving perceptual quality does not necessarily lead to better face detection or recognition. In fact, it can get even worse, and we have shown that in Section 3.

In this paper, we propose a discriminative facial feature restoring GAN which enhances the discriminative features of degraded face images. The main novelty is that it focuses on optimising figures of merit that are designed to maximally boost detection and recognition performance, while not necessarily making the images look better from a perceptual image quality standpoint. As per our knowledge, enhancement of face detection and recognition by restoring the lost features of the input images using GANs has not been explored yet. In a nutshell, our novel framework offers (a) enhancement of facial features for very low-quality images, (b) a constrained angular metric learning method that learns to reconstruct discriminative facial features(see Section 2.3), (c) and a weighted combination of different losses operating at various stages of the network to recover discriminative facial features leading to better performance. We achieve outstanding results on benchmark datasets of degraded face images (see Section $3)$.

\section{FACIAL FEATURE RESTORATION USING GAN}

\subsection{Method}

We aim to estimate a feature enhanced facial image from a low-quality image $\mathbf{I}^{G}$. Further, $\mathbf{I}^{G T}$ is the ground truth which is the feature-rich, artifact-free and clean version of $\mathbf{I}^{G}$. For an image with height $h$, width $w$ and $c$ channels, $\mathbf{I}^{G}$ and $\mathbf{I}^{G T}$ are real-valued tensors where $\mathbf{I} \in \mathbb{R}^{w \times h \times c}$. 


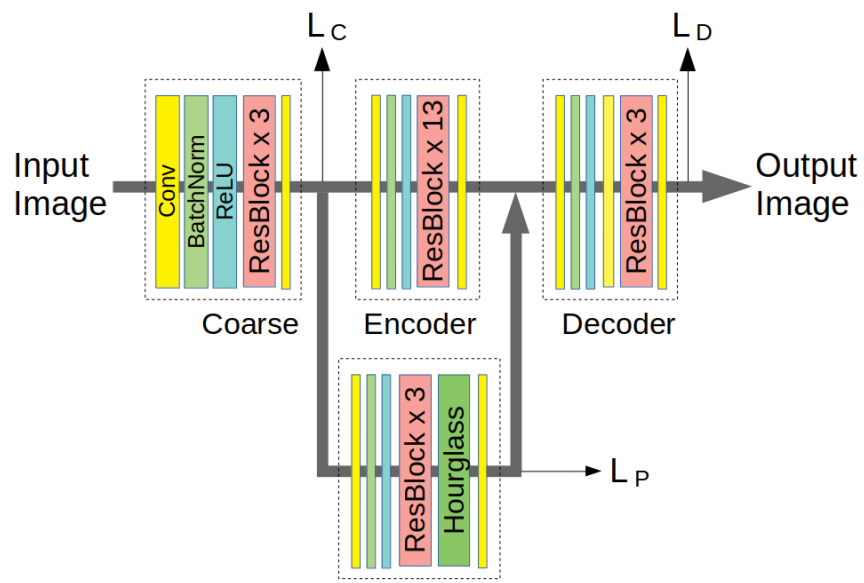

Prior Net

Fig. 1: Architecture of the generator. The ResBlock and Hourglass components are similar to [15]. $L_{C}, L_{P}$ and $L_{D}$ are explained in Section 2.4. Best viewed in colour.

In order to estimate the corresponding enhanced image, we train a generator network $G_{\theta_{G}}$ as a feed-forward CNN parameterised by $\theta_{G}$. Here $\theta_{G}=\mathbf{W}_{1: N} ; \mathbf{b}_{1: N}$ denotes the weights and biases of an N-layer deep network and is obtained by optimising a loss function $L$. The training is done using two sets of $n$ images $\left\{\mathbf{I}_{i}^{G}: i=1,2, \ldots, n\right\}$ and $\left\{\mathbf{I}_{i}^{G T}\right.$ : $i=1,2, \ldots, n\}$ such that $\mathbf{I}_{i}^{G T}=G_{\theta_{G}}\left(\mathbf{I}_{i}^{G}\right)$ (where $\mathbf{I}_{i}^{G T}$ and $\mathbf{I}_{i}^{G}$ are corresponding pairs) and our goal is to solve $\hat{\theta}_{G}=$ $\arg \min _{\theta_{G}} \frac{1}{n} \sum_{i=1}^{n} L$.

We also have a function $\Phi$ which is a facial feature extraction model, such that $\Phi(\mathbf{I}) \in \mathbb{R}^{d}$ is a $d$ dimensional facial feature vector. We also add a discriminator network [14] to evaluate the images generated by $G_{\theta_{G}}$. $G_{\theta_{G}}$ is trained to generate the target images $G_{\theta_{G}}\left(\mathbf{I}^{G}\right)$ such that the cosine distance between $\Phi\left(G_{\theta_{G}}\left(\mathbf{I}^{G}\right)\right)$ and $\Phi\left(\mathbf{I}^{G T}\right)$ tends to 0 . This is done using our proposed framework which enhances the quality while preserving the identity of the image. The generator and discriminator are trained alternatively. This adversarial min-max game encourages the generation of superior solutions residing in the manifold of feature-rich facial images.

\subsection{Network Architecture}

Figure 1 gives an overview of our generator network. This architecture is inspired by FSRNet [15] which is a superresolution network. But we are restoring degraded images keeping the resolution intact. So we modified it according to our requirements. The main changes include removal of down-sampling and up-sampling layers (apart from the hourglass block). The number of filters per convolution layer in the encoder, decoder and coarse network is 64 with $3 \times 3$ kernels and stride 1 . Furthermore, we use a single hourglass block in the prior net compared to 2 blocks in FSRNet. Our prior net is designed to reconstruct the lost facial landmarks. For the prior enhancement net, the number of filters is 128

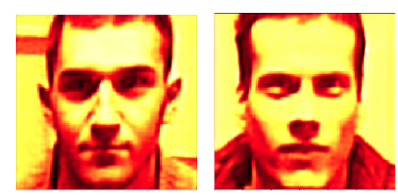

(a) Prior Network Output

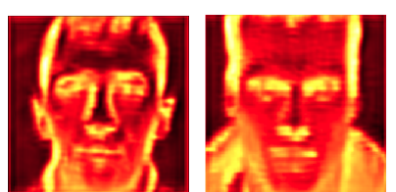

(b) Encoder Network Output
Fig. 2: Visual examples of the outputs from different network components. Best viewed in colour.

with $3 \times 3$ kernels and stride 1 except the first convolution layer where the kernel is $7 \times 7$. ReLU activation is used throughout the generator. The discriminator is in line with the architectural guidelines of the one proposed in [16].

\subsection{Constrained Angular Metric Learning of Hard and Semi-Hard Identity Features}

Inspired from [3], the constrained angular metric learning relies on two fixed points that guide the feature reconstruction in the correct direction. Let $\Phi\left(\mathbf{I}_{i}^{R}\right)$ be a reference point where $\mathbf{I}_{i}^{R}$ is the unique mugshot of identity $i$ and $i \in\{1,2, \ldots, C\}$ (considering there are $C$ classes). The mugshot is an image having clean, feature-rich, well illuminated, frontal face pose with neutral facial expression and with no occlusion. $\Phi\left(\mathbf{I}_{j}^{R}\right)$ is another reference point where $\mathbf{I}_{j}^{R}$ is the mugshot for identity $j$ where $j \in\{1,2, \ldots, C\}$ but $i \neq j$. We aim to ensure that $\Phi\left(G_{\theta_{G}}\left(\mathbf{I}_{i}^{G}\right)\right)$ is closest to $\Phi\left(\mathbf{I}_{i}^{R}\right)$. We call these similar pairs. Simultaneously $\Phi\left(G_{\theta_{G}}\left(\mathbf{I}_{i}^{G}\right)\right)$ must be as far as possible from all $\Phi\left(\mathbf{I}_{j}^{R}\right)$ and we call these dissimilar pairs. The distance between similar pairs is

$$
\begin{aligned}
& D\left(\mathbf{I}_{i}^{R}, \mathbf{I}_{i}^{G}, G_{\theta_{G}}\right) \\
& =1-\frac{\sum_{k=1}^{d} \Phi\left(\mathbf{I}_{i}^{R}\right)_{k} \Phi\left\{G_{\theta_{G}}\left(\mathbf{I}_{i}^{G}\right)\right\}_{k}}{\sqrt{\sum_{k=1}^{d} \Phi^{2}\left(\mathbf{I}_{i}^{R}\right)_{k}} \sqrt{\sum_{k=1}^{d} \Phi^{2}\left\{G_{\theta_{G}}\left(\mathbf{I}_{i}^{G}\right)\right\}_{k}}},
\end{aligned}
$$

where $k$ indexes the vector elements. Similarly, the distance between dissimilar pairs is given by $D\left(\mathbf{I}_{j}^{R}, \mathbf{I}_{i}^{G}, G_{\theta_{G}}\right)$. Training all possible 3-image sets is unnecessary and computationally expensive, thus we select a subset of 3-image sets. Each 3-image set $T_{i, j}=\left\{\mathbf{I}_{i}^{R}, G_{\theta_{G}}\left(\mathbf{I}_{i}^{G}\right), \mathbf{I}_{j}^{R}: i \neq j\right\}$, where $\Phi\left\{G_{\theta_{G}}\left(\mathbf{I}_{i}^{G}\right)\right\}$ is constrained in space by the similar and dissimilar pairs and the loss for each $T_{i, j}$

$$
L_{M E T}\left(G_{\theta_{G}}\right)=D\left(\mathbf{I}_{i}^{R}, \mathbf{I}_{i}^{G}, G_{\theta_{G}}\right)-D\left(\mathbf{I}_{j}^{R}, \mathbf{I}_{i}^{G}, G_{\theta_{G}}\right) .
$$

In an ideal scenario,

$$
D\left(\mathbf{I}_{i}^{R}, \mathbf{I}_{i}^{G}, G_{\theta_{G}}\right)<\left.D\left(\mathbf{I}_{j}^{R}, \mathbf{I}_{i}^{G}, G_{\theta_{G}}\right) \forall T_{i, j}\right|_{i \neq j}
$$

is satisfied for a trained model.

\subsection{Loss Function}

The main idea of this research is to enhance face recognition performance using the output images of the GAN and our loss 
function is designed accordingly. The components of our proposed loss function are detailed in the following sections.

\subsubsection{Identity Feature Loss}

As the preservation of identity is important for the network, the objective function needs to evaluate the identity vector of the generated images with respect to the ground truth image. Following Equation 1, the identity feature $\operatorname{loss} L_{I D}\left(G_{\theta_{G}}\right)$ is given by the angular distance

$$
L_{I D}\left(G_{\theta_{G}}\right)=D\left(\mathbf{I}_{i}^{G T}, \mathbf{I}_{i}^{G}, G_{\theta_{G}}\right) .
$$

\subsubsection{Pixel-wise Identity Loss}

Since the input images are corrupted, it is important to learn to reconstruct the corrupted images and drive the reconstruction towards the clean image manifold. This is done introducing a pixel-wise identity loss $L_{p x}$ in the cost function where

$$
L_{p x}\left(G_{\theta_{G}}\right)=\left\|\mathbf{I}_{i}^{G T}-G_{\theta_{G}}\left(\mathbf{I}_{i}^{G}\right)\right\|_{2}+\left\|\mathbf{I}_{i}^{R}-G_{\theta_{G}}\left(\mathbf{I}_{i}^{G}\right)\right\|_{2} .
$$

\subsubsection{Perceptual Loss}

The perceptual loss is the weighted sum of the content loss and the negative of the discriminator loss. To ensure a highquality output image, we choose the content loss based on the ReLU activation layers of the pre-trained 19 layer VGG[11] network. The content loss is given by $L_{V G G}\left(I_{i}^{G T}, G_{\theta_{G}}\left(\mathbf{I}_{i}^{G}\right)\right)$, which is the euclidean distance between the feature representations of a reconstructed image $\left.G_{\theta_{G}}\left(\mathbf{I}_{i}^{G}\right)\right)$ and the ground truth $\mathbf{I}_{i}^{G T}$. Details about this loss can be found in [10]. At the output of the discriminator, we use binary cross-entropy, predicting if the image is real or generated. The sigmoid of the resulting value is the discriminator loss $L_{d i s}$.

\subsubsection{Final Loss}

The final loss is a weighted sum of the cost function components with $\alpha, \beta$ and $\gamma$ as weights. To enhance prior information, we minimise the errors after the coarse network $G_{\theta_{G}}^{C}$. $L_{p x}\left(G_{\theta_{G}}^{C}\right), L_{I D}\left(G_{\theta_{G}}^{C}\right)$ and $L_{M E T}\left(G_{\theta_{G}}^{C}\right)$ is calculated at the output of the coarse network. This coarse enhancement ensures a further enhanced final image. The cost function for the coarse network is formulated as

$$
L_{C}=\beta L_{M E T}\left(G_{\theta_{G}}^{C}\right)+L_{p x}\left(G_{\theta_{G}}^{C}\right)+\alpha L_{I D}\left(G_{\theta_{G}}^{C}\right) .
$$

$L_{P}=L_{p x}\left(G_{\theta_{G}}^{P}\right)$ is calculated at the output of the prior enhancement net $G_{\theta_{G}}^{P}$ to ensure the spatial consistency of the landmarks. The loss $L_{D}$ at the output of the decoder is given by

$$
\begin{aligned}
L_{D}= & \beta L_{M E T}\left(G_{\theta_{G}}\right)+L_{p x}\left(G_{\theta_{G}}\right)+\alpha L_{I D}\left(G_{\theta_{G}}\right) \\
& +\gamma L_{V G G}\left(\mathbf{I}_{i}^{G T}, G_{\theta_{G}}\left(\mathbf{I}_{i}^{G}\right)\right)-L_{d i s} .
\end{aligned}
$$

When all the losses are combined, the final loss function $L$ is

$$
L=L_{C}+L_{P}+L_{D} \text {. }
$$

\section{EXPERIMENTS}

\subsection{Data \& Experimental Setup}

We have validated our method by testing on SCFace [17], a public benchmark dataset having low-quality face images. Our network was trained using around 1.3 million images of 14,528 identities which were obtained from the internet. The faces for training dataset were aligned and cropped using MTCNN [6]. A combination of one or more random degradation like MPEG and JPEG compression, changes in brightness and contrast, interlacing artifacts, and blurring were applied to the images in the training dataset. Some images were kept untouched to add variation to the training dataset. We trained the network on an NVIDIA DGX-Station. Each training image is $128 \times 128$ pixels. The value of $\beta, \alpha$ and $\gamma$ were empirically selected and are 250,20 and $10^{-3}$ respectively. For optimisation, we use Adam [18] with $\beta_{1}=0.9$ and a learning rate of $10^{-4}$. We use the same trained model and network for both detection and recognition experiments. Our implementation is based on TensorFlow.

\subsection{Results and Observations}

Table 1 shows a short summary of the ablation study. It highlights the importance of using a discriminator and metric learning during the training. If either of these components is removed, the performance drops massively.

Face Detection: Table 2 shows the detection performance for various algorithms, which improves by a huge margin when the images are enhanced using our method, especially for SSD+MobileNetV2 [21, 23] where there is an improvement of $27.09 \%$. Note that images enhanced using perceptual image enhancement methods [22,12,13] performs worse on detection algorithms as they do not operate on facial features but on perceptual quality which is useless for this purpose. This highlights the importance of facial feature enhancement. Face Recognition: Table 3 shows the recognition performance after image enhancement using different algorithms. The numbers in bold denote the best result. We can see in Figure 3 that the area under the ROC increases using our method, which implies a better separation between different

\begin{tabular}{l|l|lllll}
\hline \multicolumn{2}{l}{ Components } & \multicolumn{5}{c}{ TAR\% @ FAR } \\
\hline Dis & $L_{M E T}$ & $10 \%$ & $1 \%$ & $0.1 \%$ & $0.01 \%$ & $0.001 \%$ \\
\hline$\times$ & $\checkmark$ & 82.62 & 61.97 & 46.36 & 32.90 & 23.39 \\
$\checkmark$ & $\times$ & 84.62 & 63.95 & 48.74 & 31.92 & 19.47 \\
$\checkmark$ & $\checkmark$ & $\mathbf{8 8 . 0 2}$ & $\mathbf{6 7 . 9 0}$ & $\mathbf{5 1 . 0 1}$ & $\mathbf{3 9 . 1 9}$ & $\mathbf{3 3 . 3 5}$ \\
\hline
\end{tabular}

Table 1: True Accept Rate (TAR) at different False Accept Rate (FAR) with different settings for the network and loss functions on SCFace dataset. The last row shows the results of the complete framework. 


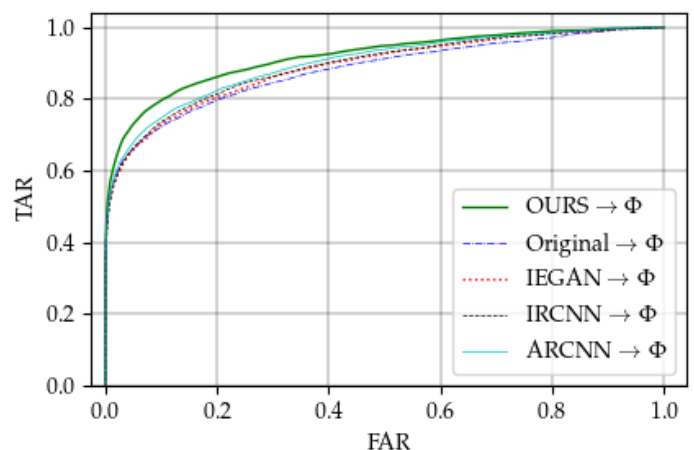

(a) $\Phi=$ Arcface [2]

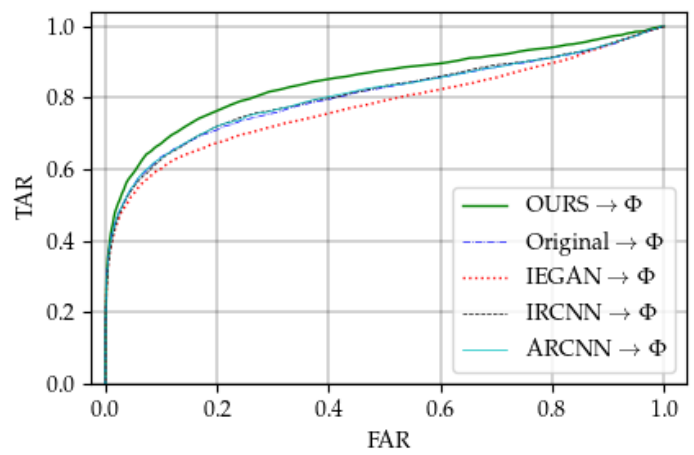

(b) $\Phi=$ CosFace [19]

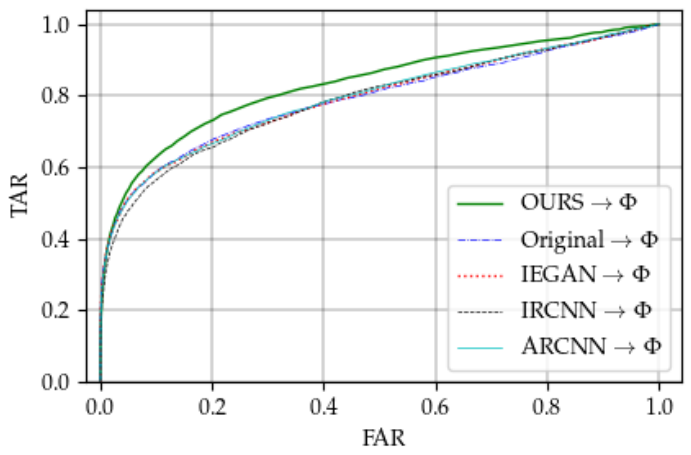

(c) $\Phi=$ FaceNet [3]

Fig. 3: ROC for different algorithms on SCFace dataset. $\mathrm{XYZ} \rightarrow \Phi$ denotes that the image was enhanced using algorithm XYZ and then recognition performance was calculated using algorithm $\Phi$. There is an improvement of $2.55 \%$ for $\mathrm{Ar}$ cFace [2], 1.95\% for CosFace [19] and 0.9\% for FaceNet [3] for the AUC after feature restoration. Best viewed in colour.

identities. This leads to a significant boost of face recognition performance for state-of-the-art face recognition algorithms. Thus the identity features of the faces at a low level are very important for superior performance on detection and recognition problems.

\section{CONCLUSION}

We presented a deep generative adversarial network for face enhancement that sets a new state-of-the-art for detection and

\begin{tabular}{c|c|c|c|c}
\hline Algorithm & dlib[20] & SSD[21] & MTCNN[6] & S3FD[1] \\
\hline Original & $90.35 \%$ & $59.27 \%$ & $91.96 \%$ & $96.01 \%$ \\
\hline ARCNN[22] & $87.38 \%$ & $55.91 \%$ & $90.94 \%$ & $96.01 \%$ \\
\hline IRCNN[12] & $87.66 \%$ & $59.09 \%$ & $90.56 \%$ & $95.84 \%$ \\
\hline IEGAN[13] & $89.02 \%$ & $50.91 \%$ & $87.76 \%$ & $92.69 \%$ \\
\hline Ours & $\mathbf{9 5 . 3 1 \%}$ & $\mathbf{8 6 . 3 6 \%}$ & $\mathbf{9 5 . 3 6 \%}$ & $\mathbf{9 9 . 0 9 \%}$ \\
\hline
\end{tabular}

Table 2: Detection rates for different algorithms on SCFace dataset for different image enhancement algorithms.

\begin{tabular}{l|lllll}
\hline Algorithm & \multicolumn{5}{|c}{ TAR\% @FAR } \\
\hline & $\mathbf{5 \%}$ & $\mathbf{1 \%}$ & $\mathbf{0 . 1 \%}$ & $\mathbf{0 . 0 1 \%}$ & $\mathbf{0 . 0 0 1 \%}$ \\
\hline ARCNN [22] & 77.39 & 65.25 & 50.62 & 38.39 & 29.72 \\
IRCNN [12] & 75.78 & 61.29 & 47.41 & 33.64 & 21.15 \\
IEGAN [13] & 76.82 & 63.09 & 48.82 & 34.71 & 20.21 \\
Ours & $\mathbf{8 2 . 0 6}$ & $\mathbf{6 7 . 9 0}$ & $\mathbf{5 1 . 0 1}$ & $\mathbf{3 9 . 1 9}$ & $\mathbf{3 3 . 3 5}$ \\
\hline
\end{tabular}

Table 3: Comparison of the recognition performance of our method with the state-of-the-art artifact and noise removal algorithms for SCFace Dataset.

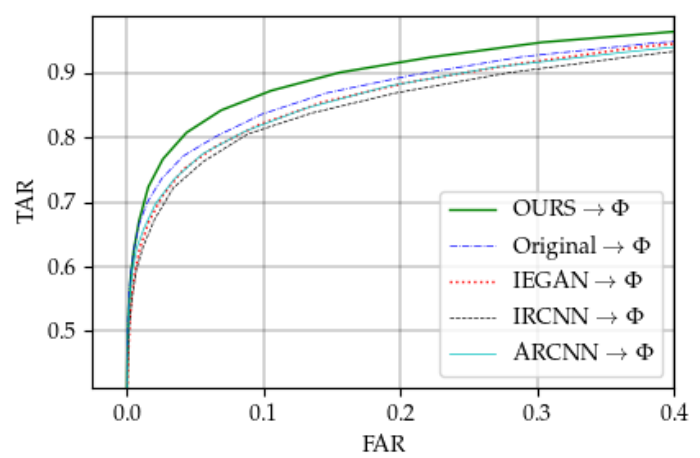

Fig. 4: A closer look at the ROC curve for different enhancement algorithms on SCFace dataset. Best viewed in colour.

recognition of low-quality images by restoring their facial features. We have highlighted some limitations of the existing algorithms and overcame those by reconstructing the facial features and increasing the separation between different classes. Specifically, we have explored the possibility of improving face recognition performance on those images for which the current state-of-the-art methods fail to deliver good results. Our proposed loss function works very well with real-world images where they inherently are of low quality. We use metric learning to train the network to generate facial images preserving their identity which is more important for improving face detection and recognition performance rather than perceptual quality, and our proposed algorithm successfully demonstrates that. 


\section{REFERENCES}

[1] Shifeng Zhang, Xiangyu Zhu, Zhen Lei, Hailin Shi, Xiaobo Wang, and Stan Z. Li, "S3fd: Single shot scaleinvariant face detector," in ICCV, 2017.

[2] Jiankang Deng, Jia Guo, Niannan Xue, and Stefanos Zafeiriou, "Arcface: Additive angular margin loss for deep face recognition," in CVPR, 2019.

[3] Florian Schroff, Dmitry Kalenichenko, and James Philbin, "Facenet: A unified embedding for face recognition and clustering," in CVPR, 2015.

[4] Y Taigman, M Yang, M Ranzato, and L Wolf, "Deepface: Closing the gap to human-level performance in face verification," in CVPR, 2014.

[5] Weiyang Liu, Yandong Wen, Zhiding Yu, Ming Li, Bhiksha Raj, and Le Song, "Sphereface: Deep hypersphere embedding for face recognition," in CVPR, 2017.

[6] K. Zhang, Z. Zhang, Z. Li, and Y. Qiao, "Joint face detection and alignment using multitask cascaded convolutional networks," IEEE Signal Processing Letters, vol. 23, no. 10, pp. 1499-1503, 2016.

[7] Joan Bruna, Pablo Sprechmann, and Yann LeCun, "Super-resolution with deep convolutional sufficient statistics," arXiv preprint arXiv:1511.05666, 2015.

[8] Alexey Dosovitskiy and Thomas Brox, "Generating images with perceptual similarity metrics based on deep networks," in NIPS, 2016.

[9] Justin Johnson, Alexandre Alahi, and Li Fei-Fei, "Perceptual losses for real-time style transfer and superresolution," in ECCV, 2016.

[10] Christian Ledig, Lucas Theis, Ferenc Huszár, Jose Caballero, Andrew Cunningham, Alejandro Acosta, Andrew Aitken, Alykhan Tejani, Johannes Totz, Zehan Wang, et al., "Photo-realistic single image superresolution using a generative adversarial network," in CVPR, 2017.

[11] Karen Simonyan and Andrew Zisserman, "Very deep convolutional networks for large-scale image recognition," in ICLR, 2015.

[12] Kai Zhang, Wangmeng Zuo, Shuhang Gu, and Lei Zhang, "Learning deep cnn denoiser prior for image restoration," in CVPR, 2017.

[13] Soumya Shubhra Ghosh, Yang Hua, Sankha Subhra Mukherjee, and Neil Robertson, "Iegan: Multi-purpose perceptual quality image enhancement using generative adversarial network," in WACV, 2019.
[14] Ian Goodfellow, Jean Pouget-Abadie, Mehdi Mirza, Bing Xu, David Warde-Farley, Sherjil Ozair, Aaron Courville, and Yoshua Bengio, "Generative adversarial nets," in NIPS, 2014.

[15] Yu Chen, Ying Tai, Xiaoming Liu, Chunhua Shen, and Jian Yang, "Fsrnet: End-to-end learning face superresolution with facial priors," in CVPR, 2018.

[16] Alec Radford, Luke Metz, and Soumith Chintala, "Unsupervised representation learning with deep convolutional generative adversarial networks," in ICLR, 2016.

[17] Mislav Grgic, Kresimir Delac, and Sonja Grgic, "Scface - surveillance cameras face database," Multimedia Tools Appl., vol. 51, pp. 863-879, 022011.

[18] Diederik P Kingma and Jimmy Ba, "Adam: A method for stochastic optimization," in ICLR, 2015.

[19] Hao Wang, Yitong Wang, Zheng Zhou, Xing Ji, Dihong Gong, Jingchao Zhou, Zhifeng Li, and Wei Liu, "Cosface: Large margin cosine loss for deep face recognition," in CVPR, 2018.

[20] Davis E. King, "Dlib-ml: A machine learning toolkit," Journal of Machine Learning Research, vol. 10, pp. 1755-1758, 2009.

[21] Wei Liu, Dragomir Anguelov, Dumitru Erhan, Christian Szegedy, Scott Reed, Cheng-Yang Fu, and Alexander C. Berg, "SSD: Single shot multibox detector," in ECCV, 2016.

[22] C. Dong, Y. Deng, C. C. Loy, and X. Tang, "Compression artifacts reduction by a deep convolutional network," in ICCV, 2015.

[23] Mark Sandler, Andrew Howard, Menglong Zhu, Andrey Zhmoginov, and Liang-Chieh Chen, "Mobilenetv2: Inverted residuals and linear bottlenecks," in CVPR, 2018. 